\title{
COMPUTATION OF THE DIFFRACTION TRANSFER MATRIX AND THE RADIATION CHARACTERISTICS IN THE OPEN-SOURCE BEM CODE NEMOH
}

\author{
Francesc Fàbregas Flavià $^{\mathrm{a}}, \quad$ Cameron McNatt $^{\mathrm{b}}, \quad$ François Rongère $^{\mathrm{a}}$ \\ Aurélien Babarit ${ }^{\text {a }}, \quad$ Alain H. Clément ${ }^{\text {a }}$ \\ a Ecole Centrale de Nantes, LHEEA \\ b The University of Edinburgh, Institute for Energy Systems \\ UMR CNRS 6598 - Nantes, France \\ School of Engineering - Edinburgh, UK
}

\begin{abstract}
Until now, widely available boundary element method (BEM) codes did not allow the calculation of two nonconventional hydrodynamic operators, which characterize the way a body diffracts and radiates waves, known as Diffraction Transfer Matrix and Radiation Characteristics respectively. When embedded into the finite-depth interaction theory developed by [1], they drastically speed up the computation of the added mass, damping and excitation force coefficients of a group ("farm") of floating bodies.

This paper presents the implementation of their computation in the open source BEM solver NEMOH using the methodology proposed by [2]. Results for two different geometries, a cylinder and a square box, are presented and compared to an alternative computational approach developed by [3]. A very good agreement between them is found. In addition, the hydrodynamic operators of the cylinder are compared to a semi-analytical solution available in the literature showing a good match. Results obtained using the finite-depth interaction theory are shown for a generic multi-body wave energy converter (WEC) demonstrating how the capabilities added to the BEM software NEMOH can facilitate the numerical modeling of the hydrodynamic interactions in large arrays of bodies.
\end{abstract}

\section{INTRODUCTION}

Due to the limited rated power capacity of the present WEC technologies, it is nowadays well-accepted that commercial exploitation of wave energy involves the installation of a large number of wave energy converters (WECs) in an array. It has been shown that radiation and scattering of waves can affect the to- tal energy output of the devices in different ways depending on the layout. The well-known radiation and excitation force coefficients contain the required information on hydrodynamic interactions and, therefore, their calculation is of paramount importance. Notwithstanding, their direct computation for a large number of bodies is beyond the capabilities of widely available standard boundary element method (BEM) codes.

The methodology developed by [1] enables one to circumvent such limitation and accelerate the computation of the hydrodynamic coefficients in finite-depth for cases where a large number of bodies is present. It is based on knowing how an individual isolated device scatters and radiates waves. For this, two hydrodynamic operators known as Diffraction Transfer Matrix (DTM) and Radiation Characteristics (RC) need to be computed. The methodology to obtain them for axisymmetric bodies was provided by [1]. The generalization of the method was developed by [2] who used an approach based on a cylindrical representation of the Green's function to calculate the elements of the DTM and $\mathrm{RC}$ for an arbitrary geometry using a BEM.

The methodology of [2] was used to study the forces on the fixed [4] and floating [5] modules of an interconnected multimoduled floating offshore structure used by the US Navy. It was also employed by [6] to study the interactions between ocean waves and large fields of ice floes in the marginal ice zone. For that, the extension of the theory to infinite-depth was required. Based on [1], [7] derived a hierarchical interaction theory aimed at studying hydrodynamic interactions among a great number of bodies in very large floating structures.

The methodology of [2] requires the modification of the diffraction problem boundary conditions, as well as access to the source strength distribution on the discretized wetted surface of 
the body. This output is not accessible in the majority of widely available standard BEM codes which only provide the standard hydrodynamic excitation forces and radiation coefficients. Because of that, [3] developed and validated an alternative approach to the one of [2] to calculate the DTM and RC using the standard output of available BEM codes.

The release in 2014 of the open source BEM solver $\mathrm{NEMOH}$, developed at Ecole Centrale de Nantes (ECN) for more than 30 years, opened the possibility of embedding the methodology of [2] into it. This paper presents the details of its implementation, validation and the comparison of results with the alternative computational approach developed by [3]. It is expected that the ease of calculation of the DTM and RC by NEMOH will facilitate the use of the interaction theory in applications such as the optimization of offshore wave farms.

\section{THEORY}

The Direct Matrix Method interaction theory (IT) [1] is based on the same hypothesis as the linear potential flow theory [8], i.e., linearity and ideal fluid characteristics. The former applies as long as a small wave steepness and a small amplitude of the body motions with respect to its characteristic dimension can be assumed. The latter holds if the fluid can be characterized as inviscid, irrotational and incompressible. In this case all the flow engineering quantities of interest can be derived from a scalar vector field known as velocity potential $\Phi$. If in addition to the previous hypothesis, an harmonic time dependence is adopted, the spatial and time variation of $\Phi$ can be decoupled as $\Phi=\operatorname{Re}\left\{\phi(x, y, z) e^{-i \omega t}\right\}$, where $\Phi$ is the velocity potential in the domain, $\phi$ is its complex spatial part, $(x, y, z)$ the spatial coordinates in a global Cartesian reference system, $i=\sqrt{-1}, \omega$ the pulsation and $t$ the time. The notation of this paper will follow the work of [1].

Because of the linearity of the problem, the total potential $\phi_{j}$ can be decomposed into $\phi_{j}^{I}+\phi_{j}^{S}+\sum_{k=1}^{N_{j}} \phi_{j}^{R k}$, where subindex $j$ refers to the body, superindices $I, S$ and $R$ refer respectively to incident, scattered and radiated potentials and $N_{j}$ stands for the number of degrees of freedom $k$ of body $j$.

\section{Partial Waves}

In a large array, waves emanating from each body (due to scattering and radiation) will propagate and interact with its neighbours. This will lead to a succession of scattering events which are referred to as multiple-scattering problem [9]. In this context, the representation of the scattered potential by a body $j$

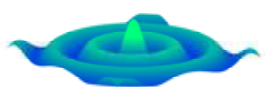

(a) $m=0, n=0$

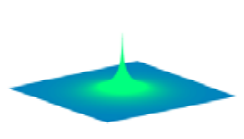

(d) $m=0, n=1$

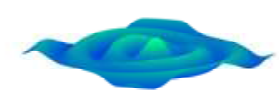

(b) $|m|=1, n=0$

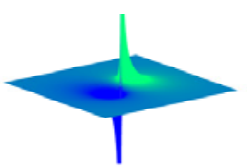

(e) $|m|=1, n=1$

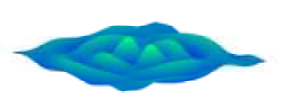

(c) $|m|=2, n=0$

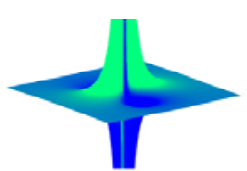

(f) $|m|=2, n=1$
FIGURE 1: Partial waves modes. Progressive term $\operatorname{Re}\left\{H_{m}^{(1)}(r)\right\}$ (a, b, c); evanescent term $\operatorname{Re}\left\{K_{m}(r)\right\}(\mathrm{d}, \mathrm{e}, \mathrm{f})$

follows as:

$$
\begin{aligned}
\phi_{j}^{S}=\sum_{m=-\infty}^{\infty}[ & \left(A_{j}^{S}\right)_{0 m} \frac{\cosh k_{0}\left(z_{j}+d\right)}{\cosh k_{0} d} H_{m}^{(1)}\left(k_{0} r_{j}\right) \\
& \left.+\sum_{n=1}^{\infty}\left(A_{j}^{S}\right)_{n m} \cos k_{n}\left(z_{j}+d\right) K_{m}\left(k_{n} r_{j}\right)\right] e^{i m \theta_{j}}
\end{aligned}
$$

where $H_{m}^{(1)}$ is the Hankel function of the first kind of order $m$, $K_{m}$ is the modified Bessel function of the second kind of order $m,\left(A_{j}^{S}\right)_{n m}$ are scattered complex coefficients, subindices $m$ and $n$ are the modes representing the angular and depth variation of the scattered potential respectively, $d$ is the water depth, $\left(z_{j}, r_{j}, \theta_{j}\right)$ are the cylindrical coordinates local to body $j$ and $k_{0}$ and $k_{n}$ represent the progressive and evanescent wave numbers obtained from the dispersion equations of the linear water wave theory.

The potential in (1) represents a superposition of partial cylindrical waves (Figure 1) which are more suited to express the disturbances on the wave field caused by the finite-sized floating bodies than the conventional long-crested plane waves [10]. In fact, the latter can also be expressed as a summation of partial cylindrical waves. For convenience, the different forms of the potential (incident, scattered and radiated) are represented as the scalar product between an infinite vector of complex coefficients and a vector of partial cylindrical wave components which are expressed relative to the local reference system of body $j$ :

$$
\phi_{j}^{S}=\left(A_{j}^{S}\right)^{T} \psi_{j}^{S} ; \quad \phi_{j}^{R}=\left(R_{j}^{k}\right)^{T} \psi_{j}^{S} ; \quad \phi_{j}^{I}=\left(A_{j}^{I}\right)^{T} \psi_{j}^{I}
$$

where $T$ stands for transposed, $A_{j}^{S}, A_{j}^{I}$ and $R_{j}^{k}$ are the complex scattered, incident and radiated vectors of partial waves coefficients whose elements represent an $(n, m)$ or $(l, q)$ index pair. For clarity of notation, indexs $(n, m)$ are associated with outgoing waves and $(l, q)$ with incident waves. $\psi_{j}^{S}$ and $\psi_{j}^{I}$ are the vectors 
of scattered and incident partial cylindrical functions which depend on the radial coordinates of the problem and the frequency.

The first part $(n, l=0)$ of the summation in (1) represents partial progressive waves, i.e., waves that propagate away from the body (Figures 1a, $1 \mathrm{~b}$ and $1 \mathrm{c})$. The second part $(n, l \neq 0)$ represents standing waves known as evanescent modes which fit the far-field potential to the body geometry (Figures 1d, 1e and 1f). In an ambient plane incident wave, no evanescent modes are present.

Even though the vectors in (2) are theoretically infinitely long, for practical computations they need to be truncated. Their dimension is given as $(2 M+1) \cdot(N+1)$ where the summations go from $m=-M$ to $M$ and from $n=0$ to $N$ (the same expression can be applied for modes $q$ and $l$ with truncation limits $Q$ and $L$ respectively). Further details on the derivation of cylindrical partial waves can be found in $[10,11]$.

\section{Interaction Theory}

The case where a plane long-crested incident wave acts upon an array of $N_{b}$ fixed bodies is considered to exemplify the use of the IT by [1]. For a more detailed description see $[1,3,5]$. One of the key aspects of the method is that the study of the wave scattering on the whole array is undertaken by focusing on one body at a time. This body is referred to as $j$ and its neighbours as $i$. The total incident potential to $j$, which can be expressed in the form $\phi_{j}^{I}=\left(A_{j}^{I}\right)^{T} \psi_{j}^{I}$, is composed of the ambient incident wave as well as the unknown scattered potentials by all the neighbouring bodies $i$ :

$$
\phi_{j}^{I}=\left(a_{j}^{T}+\sum_{\substack{i=1 \\ i \neq j}}^{N_{b}} A_{i}^{T} \mathbf{T}_{i j}\right) \psi_{j}^{S}
$$

where $a_{j}$ are the ambient plane wave cylindrical coefficients, $A_{i}$ are unknown scattered coefficients and $\mathbf{T}_{i j}$ represents the transformation matrix which depends on the relative position between bodies $j$ and $i$. The latter enables one to express scattered waves from a body $i$ as incident to body $j$ in its local reference system.

The incident and scattered partial waves coefficients by an isolated body can be related by means of a linear operator known as Diffraction Transfer Matrix $\left(\mathbf{B}_{j}\right)$ :

$$
A_{j}^{S}=\mathbf{B}_{j} A_{j}^{I}
$$

The elements $\left(\mathbf{B}_{j}\right)_{n l}^{m q}$ are defined as the coefficient of the partial wave of depth mode $n$ and angular mode $m$ in the scattered potential in response to a unit incident wave of depth mode $l$ and angular mode $q$ [1]. By substituting the incident partial coefficients in (3) into (4), the scattered coefficients due to incident waves $A_{j}^{I}$ to body $j$ are:

$$
A_{j}^{S}=\mathbf{B}_{j}\left(a_{j}+\sum_{\substack{i=1 \\ i \neq j}}^{N_{b}} \mathbf{T}_{i j}^{T} A_{i}\right)
$$

If the same procedure that has been applied so far to body $j$ is repeated for the rest of devices in the array, as many expressions of the form (5) as bodies $\left(N_{b}\right)$ will be obtained. The system can then be solved for the unknown scattered coefficients enabling one to reconstruct the total potential in the domain and to compute quantities of interest such as the forces exerted on the bodies. In that sense, [3] introduced a linear operator called Force Transfer Matrix which facilitates this operation.

The example described corresponds to a diffraction problem. The same procedure could be applied to solve a radiation problem where a body $i$ of the array undergoes a motion in a degree of freedom $k$. In this case, the vector of ambient incident waves to body $j,\left(a_{j}\right)$, should be substituted by the radiated partial wave coefficients $R_{i}^{k}$ (known as Radiation Characteristics) expressed in the local coordinate system of body $j$ as $\mathbf{T}_{i j}^{T} R_{i}^{k}$. Further details on the method can be found in $[2,3,5]$.

Before being able to solve the system in (5) it is necessary to determine the Diffraction Transfer Matrix (DTM), as well as the Radiation Characteristics (RC) if the body in the array is undergoing motion. Both operators are calculated with the body in isolation and depend on its geometry and the frequency $(\omega)$. In addition, the Radiation Characteristics depend on the mode of motion. Two methodologies to compute them [2,3] have been identified in the literature. The former, which has been implemented in NEMOH will be referred to as Method 1, whereas the latter to which the results will be compared as Method 2 .

\section{Method 1}

\section{Diffraction Transfer Matrix}

The methodology developed by [2] enables one to find each element of the diffraction transfer matrix $\left(\mathbf{B}_{j}\right)_{n l}^{m q}$ of an isolated body $j$ following two steps. First, the solution to a diffraction problem where the incident wave is a cylindrical partial wave of angular mode $q$ and depth mode $l$ is found. Once the source strength distribution $\sigma_{l q j}$ known, the following step consists of expressing the scattered potential in the base of partial wave functions (1). This is to be achieved by making use of the Green's function in cylindrical coordinates developed by [12] which leads to the following expressions for the elements of the Diffraction 
Transfer Matrix:

$$
\begin{aligned}
& B_{0 m j}=\frac{i}{2} C_{0} \cosh k_{0} d \times \\
& \iint_{S_{H j}} \sigma_{l q j}\left(R_{j}, \Theta_{j}, \zeta_{j}\right) J_{m}\left(k_{0} R_{j}\right) \cosh \left[k_{0}\left(\zeta_{j}+d\right)\right] e^{-i m \Theta_{j}} d s \\
& B_{n m j}=-\frac{1}{\pi} C_{n} \times \\
& \quad \iint_{S_{H j}} \sigma_{l q j}\left(R_{j}, \Theta_{j}, \zeta_{j}\right) I_{m}\left(k_{n} R_{j}\right) \cos \left[k_{n}\left(\zeta_{j}+d\right)\right] e^{-i m \Theta_{j}} d s
\end{aligned}
$$

where $J_{m}$ is the Bessel function of the first kind of order $m, I_{m}$ is the modified Bessel function of the first kind of order $m, C_{0}$ and $C_{n}$ are constant coefficients and $S_{H j}$ is the wetted surface of body $j$.

\section{Radiation Characteristics}

The same principle applied for the calculation of the DTM can be used to obtain the RC vector. First, the radiation problem associated with a motion mode $k$ of the body under consideration is solved. Once known, the source strength distribution $\sigma_{j k}$ is used in conjunction with the Green's function in cylindrical coordinates to express the radiated potential in the base of partial waves leading to expressions (8) and (9) for the radiation characteristics:

$$
\begin{aligned}
& R_{0 m j}=\frac{i}{2} C_{0} \cosh k_{0} d \times \\
& \iint_{S_{H j}} \sigma_{j k}\left(R_{j}, \Theta_{j}, \zeta_{j}\right) J_{m}\left(k_{0} R_{j}\right) \cosh \left[k_{0}\left(\zeta_{j}+d\right)\right] e^{-i m \Theta_{j}} d s \\
& R_{n m j}=-\frac{1}{\pi} C_{n} \times \\
& \quad \iint_{S_{H j}} \sigma_{j k}\left(R_{j}, \Theta_{j}, \zeta_{j}\right) I_{m}\left(k_{n} R_{j}\right) \cos \left[k_{n}\left(\zeta_{j}+d\right)\right] e^{-i m \Theta_{j}} d s
\end{aligned}
$$

\section{Method 2}

\section{Diffraction Transfer Matrix}

The methodology by [2] involves solving the scattering BVP using incident partial cylindrical waves. This capability was not included in standard BEM codes until now that has been made available in NEMOH. A procedure based on the DTM definition was derived by [3] to circumvent this need and calculate its elements by solving several diffraction problems requiring only plane incident waves.

Equation (4) can be transformed into a system of equations to solve for the elements of the DTM as long as a large enough number of pairs of scattered/incident vectors of coefficients is known in advance. This is to be achieved by solving, at least, as many diffraction problems as the leading dimension of the DTM by changing at each time the propagation direction $(\beta)$ of the plane incident wave. The vectors of incident partial waves are known from an analytical expression [2] whereas the elements of the associated vectors of the scattered coefficients can be derived by means of a Fourier Transform of the scattered potential on the body circumscribing cylinder:

$$
\begin{array}{r}
a_{0 m}^{S}=-\frac{i}{2 \pi} \frac{\omega}{g} \frac{2 \cosh k_{0} d}{d\left(1+\frac{\sinh 2 k_{0} d}{2 k_{0} d}\right)} \frac{1}{H_{m}^{(2)}\left(k_{0} r_{0}\right)} \times \\
\int_{-d}^{0} \int_{0}^{2 \pi} \phi\left(r_{0}, \theta, z\right) e^{-i m \theta} \cosh k_{0}(d+z) d \theta d z \\
b_{n m}^{S}=-\frac{i}{2 \pi} \frac{\omega}{g} \frac{2}{d\left(1+\frac{\sin 2 k_{n} d}{2 k_{n} d}\right)} \frac{1}{K_{m}\left(k_{n} r_{0}\right)} \times \\
\int_{-d}^{0} \int_{0}^{2 \pi} \phi\left(r_{0}, \theta, z\right) e^{-i m \theta} \cos k_{n}(d+z) d \theta d z
\end{array}
$$

where $\left(r_{0}, \theta, z\right)$ are the coordinates of the points on a circumscribing cylindrical control surface.

Even if evanescent terms from the scattered potential can be identified using (11), the use of only plane progressive incident waves (with no evanescent components) prevents the calculation of the DTM terms relating incident and scattered evanescent partial waves using Method 2.

\section{Radiation Characteristics}

The computation of the RC follows the same procedure as for the DTM. First, the radiation problem associated with a specific mode of motion and frequency is solved with a standard BEM code. Then, by means of expressions (10) and (11), the radiated potential is expressed in terms of partial cylindrical wave functions. In this base, the coefficients are known as Radiation Characteristics.

\section{Equivalence between Methods 1 and 2}

The equivalence of the DTM elements between both methodologies, which make use of different notation conventions and a different scaling of the partial wave coefficients, is given by:

$$
\frac{(-1)^{-m}}{(-1)^{-q}}\left(\mathbf{B}^{*}\right)_{-m,-q}^{\operatorname{Method} 1}=(\mathbf{B})_{m, q}^{\operatorname{Method} 2}
$$

In addition, the use of two different BEM solvers (Method 1 has been implemented in NEMOH whereas WAMIT has been used 
in conjunction with Method 2) results in the following relationship between the Radiation Characteristics:

$$
(-1)^{-m} \frac{g}{\omega^{2}}\left[\left(a_{-m k}^{R}\right)\right]^{*}=R_{m k}
$$

where $a_{-m k}^{R}$ are the RC in the notation of [3] and $R_{m k}$ in the notation of [2].

\section{NUMERICAL IMPLEMENTATION}

As mentioned in [13], the definition of the body boundary conditions in NEMOH is flexible. Thus, for the diffraction problem, the BEM code can easily accommodate a user-defined distribution of normal velocities at the center of gravity of each mesh panel. They have been implemented in the Pre-Processor module as the derivative of the incident partial wave functions (of angular mode $q$ and depth mode $l$ ) whose full expression can be found in [2].

The integrals (6) - (9) cannot be solved analytically as there exists no closed mathematical expression for the source strengths. Therefore, they need to be solved using a quadrature scheme. In this study, results will be presented for a one-point and four-point Gaussian quadrature rules [14] which have been implemented in the NEMOH Post-Processor module.

\section{RESULTS}

In this section, the main components of the Diffraction Transfer Matrix and the Radiation Characteristics for a truncated vertical cylinder and a square box are presented. The choice of two different bodies enables one to identify geometry specific features of the hydrodynamic operators. The discretizations shown in Figures $2 \mathrm{a}$ and $3 \mathrm{a}$ have been used for the computations. A mesh convergence study has been performed showing no significant improvement of accuracy by using $2 \mathrm{~b}$ and $3 \mathrm{~b}$. In addition, results using the interaction theory by [1] into which the calculated DTM and RC have been embedded are shown for a small array of two truncated vertical cylinders.

\section{Diffraction Transfer Matrix \\ Cylinder}

Figures $4 \mathrm{a}$ and $4 \mathrm{~b}$ show the real and imaginary parts of the cylinder DTM components. For clarity, only progressive terms are shown. A very good agreement between results obtained using Method 1 (with both one-point and four-point quadrature schemes), Method 2 and the semi-analytical solution by [15] is found. It can be observed that the only non-zero DTM terms correspond to pairs of equal incident $(q)$ and outgoing $(m)$ angular modes. This is a particular feature of axisymmetric geometries such as the truncated vertical cylinder. In addition, it can

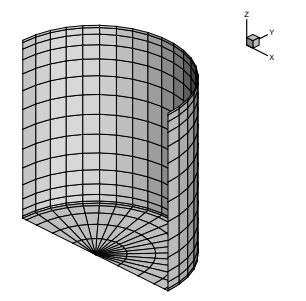

(a) Number of panels: 361

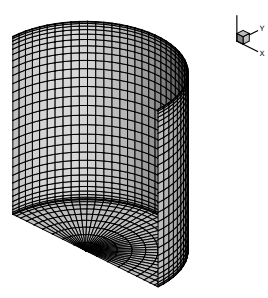

(b) Number of panels: 1521
FIGURE 2: Truncated vertical cylinder mesh. Only half of the geometry is shown due to symmetry.

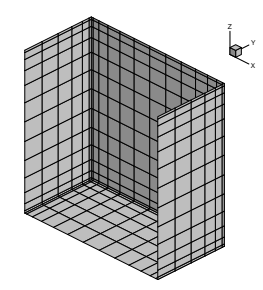

(a) Number of panels: 403

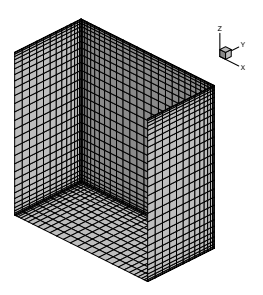

(b) Number of panels: 2059
FIGURE 3: Square box mesh. Only half of the geometry is shown due to symmetry.

be appreciated that the number of significant angular modes is different for each frequency. For instance, a truncation of only two angular modes is sufficient at $k a \approx 1$ but not at $k a \approx 2$ where three angular modes are required.

The numerical singularity observed at a $k a$ of approximately 2.3 corresponds to an irregular frequency. The new release of the BEM solver NEMOH will enable its removal. As mentioned in [13] they are generally high frequencies and in this case they fall out of the range of interest for practical wave energy converter applications. The wide range of $k a$ values checked is aimed at increasing the robustness and reliability of the methodology implemented.

\section{Square Box}

Figures $5 \mathrm{a}$ and $5 \mathrm{~b}$ show the real and imaginary parts of the square box DTM components. Similar to the truncated vertical cylinder, good agreement between the results obtained using Method 1 and 2 can be observed. For this non-axisymmetric geometry, terms of the DTM involving different incident and outgoing angular modes, such as the pair $(q=1, m=-3)$, are non-zero. In addition, it can be appreciated that for a wide range of $k a$ values the angular mode truncation is higher than for the truncated vertical cylinder. No improvement of results is obtained whether using a one-point or four-point quadrature integration scheme. 


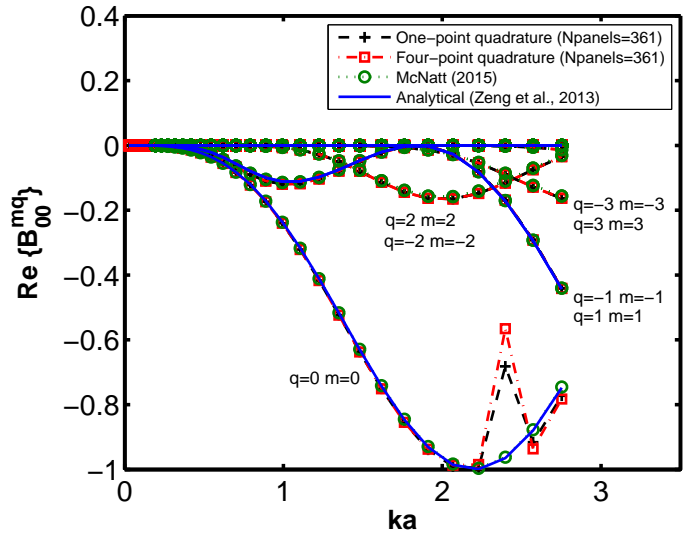

(a)

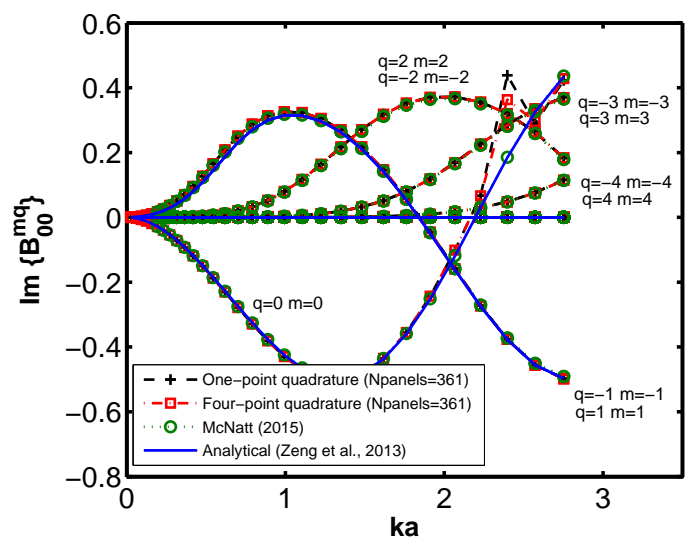

(b)

FIGURE 4: Real and imaginary parts of the Diffraction Transfer Matrix progressive terms for a truncated vertical cylinder of $3 m$ radius $(a), 6 m$ draft in a $10 m$ water depth.

\section{Radiation Characteristics}

\section{Cylinder}

The progressive terms of the surge Radiation Characteristics of the cylinder are shown in Figure 6. Good agreement between both methods and the semi-analytical solution can be observed, as well as no significant differences between integration schemes. For this mode of motion, it can be observed that only modes $m=1$ and $m=-1$ are non-zero. This is explained as the wave generated by the motion of a cylinder in surge corresponds to the partial wave shown in Figure 1b. For heave (Figure 8), only the mode $m=0$, representing an isotropic wave (1a) is required [10]. More discrepancy of results is observed in the real part compared to the imaginary whose magnitude is significantly higher than the former. It is hypothesized that the differences in angular mode truncation among methods could affect the radiated energy distribution on the outgoing partial

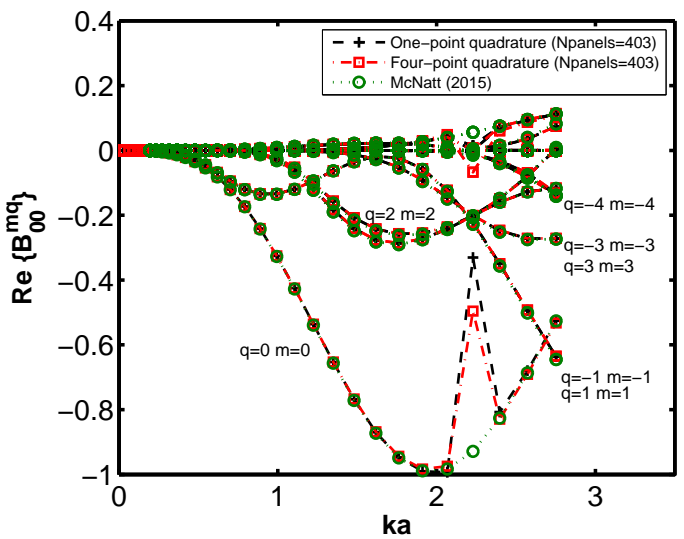

(a)

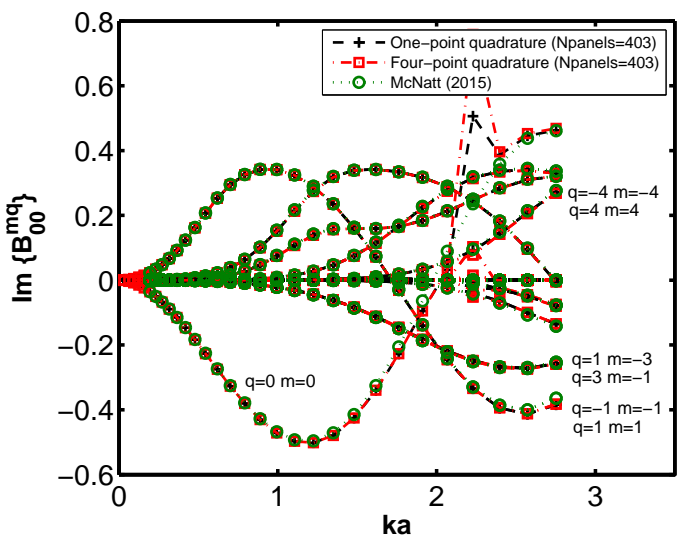

(b)

FIGURE 5: Real and imaginary parts of the Diffraction Transfer Matrix progressive terms for a square box of $6 m$ side $(2 a), 6 m$ draft in a $10 m$ water depth.

wave functions basis and be the cause of the disagreement. For this mode of motion, the presence of an irregular frequency at the same $k a$ value as for the DTM can be observed.

\section{Square Box}

Compared to the cylinder, additional angular modes of order three appear in the Radiation Characteristics of the square box moving in surge. The radiated field for this geometry and for this mode of motion is more complex than the one generated by the truncated vertical cylinder and a superposition of partial waves is required to represent it. For heave (Figure 9), only the isotropic partial wave $(m=0)$ is needed.

\section{Interaction Theory}

Results for the hydrodynamic coefficients obtained using the interaction theory by [1] are shown in Figure 10 for a small array 
TABLE 1: Interaction coefficients of the diffraction problem $(\beta=0)$ for the first cylinder of the array in Figure $9(\lambda / a=5)$

\begin{tabular}{c|ccccc|ccccc}
\hline $\mathrm{A}$ & \multicolumn{6}{|c|}{$\mathrm{d} / \mathrm{a}=10$} & \multicolumn{3}{c}{$\mathrm{d} / \mathrm{a}=6$} \\
\hline $\mathrm{m}, \mathrm{n}$ & Analytical & IT coarse & IT fine & Coarse $(\%)$ & Fine $(\%)$ & Analytical & IT coarse & IT fine & Coarse $(\%)$ & Fine $(\%)$ \\
\hline$-1,0$ & $(0.7604,-1.5687)$ & $(0.7835,-1.5901)$ & $(0.7657,-1.5756)$ & $(3.0,1.4)$ & $(0.7,0.4)$ & $(0.2912,-1.0623)$ & $(0.2988,-1.0758)$ & $(0.2940,-1.0649)$ & $(2.6,1.3)$ & $(1.0,0.2)$ \\
0,0 & $(-1.6607,2.1404)$ & $(-1.6565,2.1857)$ & $(-1.6606,2.1735)$ & $(0.3,2.1)$ & $(0.0,1.5)$ & $(-3.0601,2.6367)$ & $(-3.0829,2.6994)$ & $(-3.0753,2.6902)$ & $(0.7,2.4)$ & $(0.5,2.0)$ \\
1,0 & $(-0.7604,1.5687)$ & $(-0.7835,1.5901)$ & $(-0.7657,1.5756)$ & $(3.0,1.4)$ & $(0.7,0.4)$ & $(-0.2912,1.0623)$ & $(-0.2988,1.0758)$ & $(-0.2940,1.0649)$ & $(2.6,1.3)$ & $(1.0,0.2)$ \\
\hline
\end{tabular}

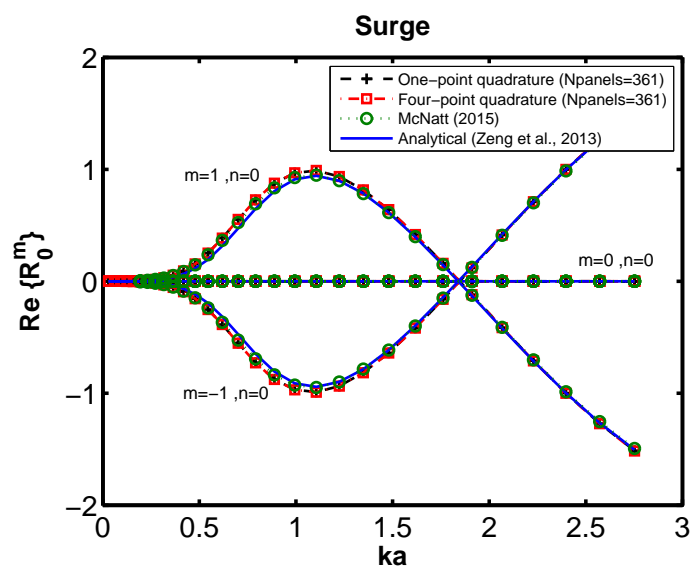

(a)

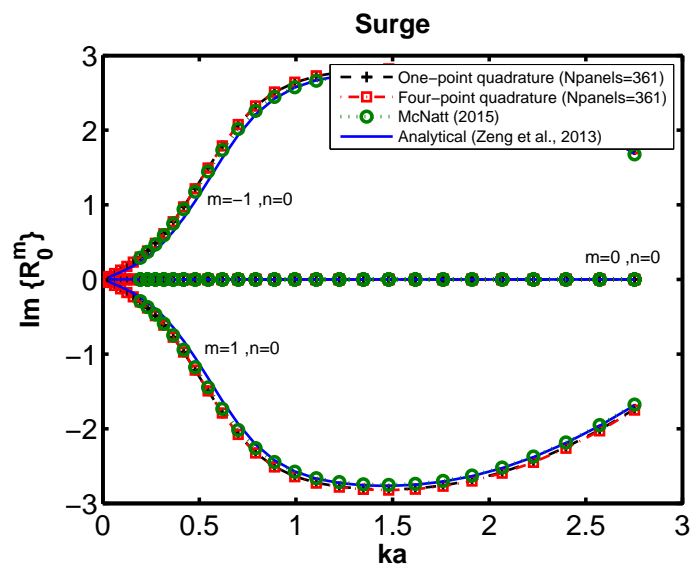

(b)

FIGURE 6: Real and imaginary parts of the Radiation Characteristics progressive terms for a truncated vertical cylinder of $3 m$ radius $(a), 6 m$ draft moving in surge in a $10 m$ water depth.

of two cylinders of $3 m$ radius $(a), 6 m$ draft in a $50 m$ water depth (Figure 11). They are compared to direct calculations performed with the BEM solver NEMOH. Several separating distance cases are considered. When only a single line is visible, it means that the other lines lie underneath it and there is a perfect match.

First, both bodies are set far away from each other at a distance corresponding to $d / a=200$. As expected, the hydrody-

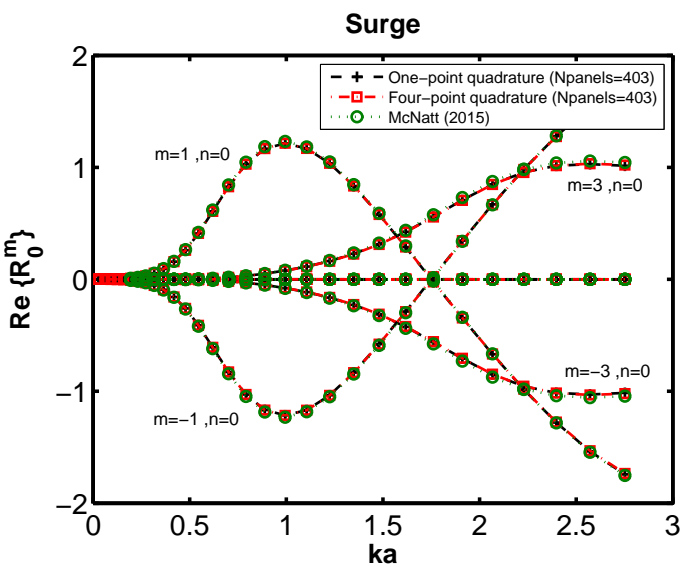

(a)

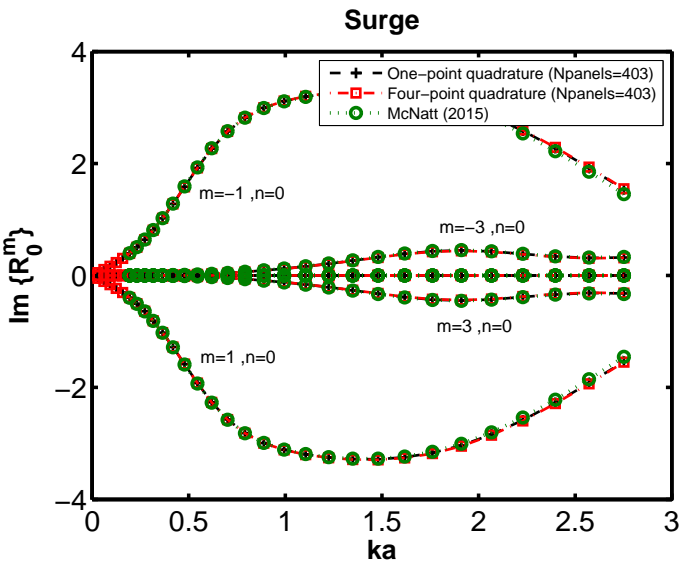

(b)

FIGURE 7: Real and imaginary parts of the Radiation Characteristics progressive terms for a square box of $6 \mathrm{~m}$ side $(2 a), 6 \mathrm{~m}$ draft moving in surge in a $10 \mathrm{~m}$ water depth.

namic coefficients are the same as if the cylinders were in isolation. Moreover, for such a long separating distance the evanescent modes do not play an important role. This can be derived from the fact that a truncation with $L=0$ leads to a perfect match of results between the IT and the direct computations using NEMOH (Figure 10a).

When bodies are set closer, in this case at a distance of 


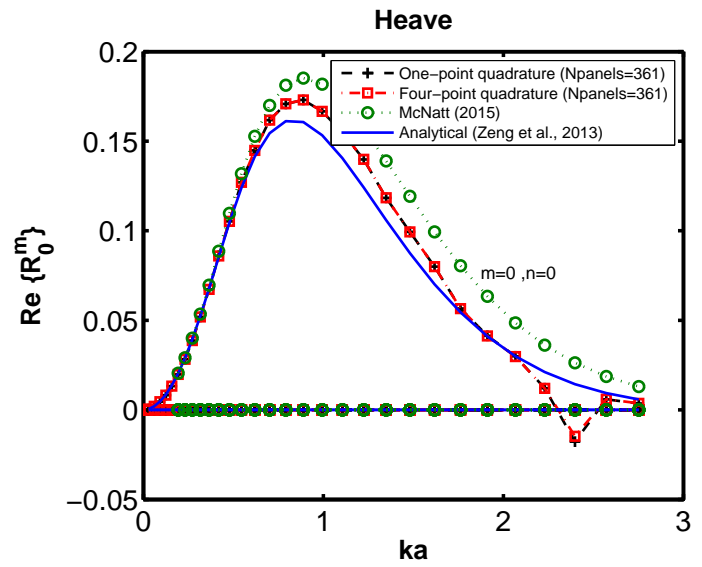

(a)

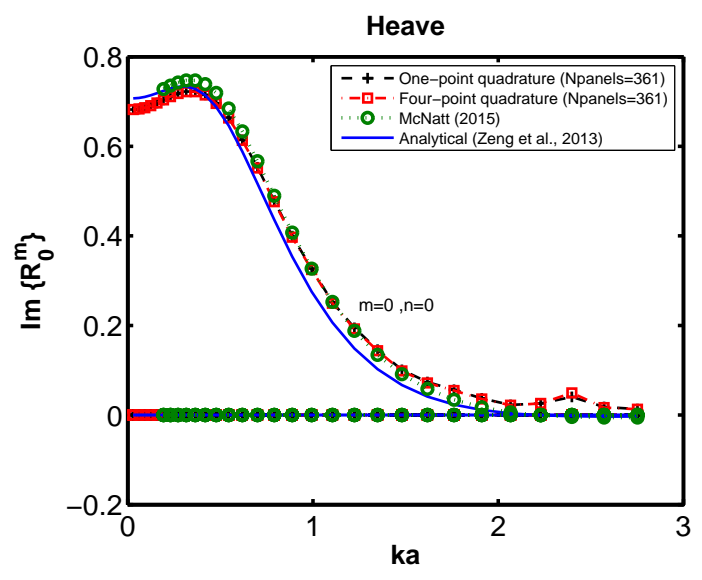

(b)

FIGURE 8: Real and imaginary parts of the Radiation Characteristics progressive terms for a truncated vertical cylinder of $3 m$ radius $(a), 6 m$ draft moving in heave in a $10 m$ water depth.

$d / a=10$, hydrodynamic interactions become important as can be observed in Figures 10b and 10c. For frequencies $\omega>1 \mathrm{rad} / \mathrm{s}$, the added mass and damping coefficients in isolation are altered. However, the influence of the evanescent modes is still negligible.

Finally, for a fixed frequency corresponding to a wave length of $(\lambda / a=10)$, the coupling heave-heave added mass coefficient $(3,9)$ is shown (Figure 12) at several separating distances which span from the previous $d / a=10$ to the limit case where the perimeters of both cylinders are externally tangent $(d / a=2)$. The influence of the evanescent modes for the heave-heave coupling can be clearly observed, as well as convergence of the IT results to the NEMOH direct calculation when the truncation of evanescent modes is increased. It has been observed that their importance to ensure accuracy of the radiation hydrodynamic coefficients for close separating distances is mainly dependent on

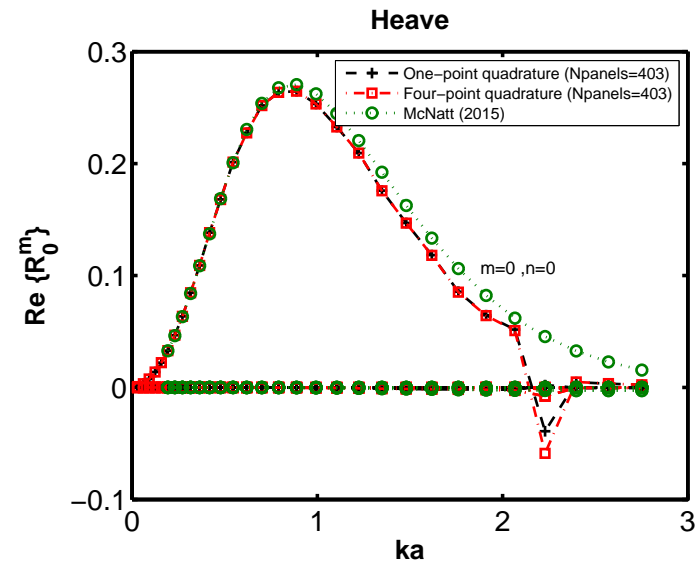

(a)

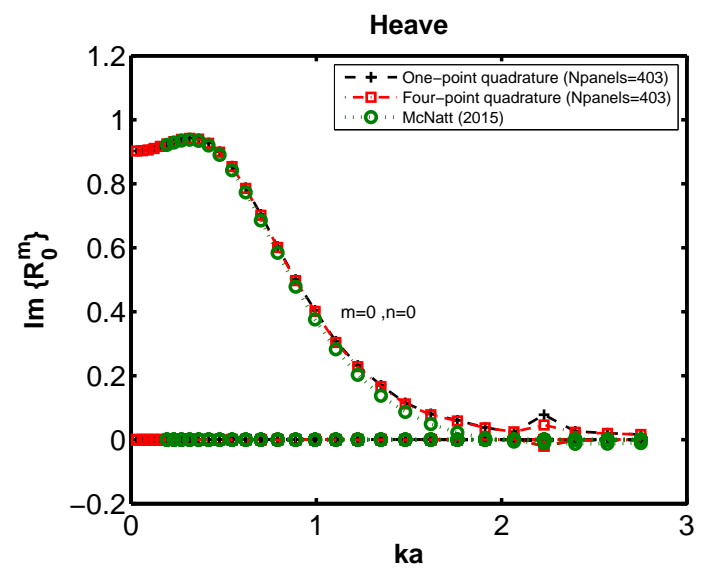

(b)

FIGURE 9: Real and imaginary parts of the Radiation Characteristics progressive terms for a square box of $6 m$ side $(2 a), 6 m$ draft moving in heave in a $10 \mathrm{~m}$ water depth.

the motion mode and the frequency. For instance, for the surgesurge coupling, their influence at $\lambda / a=10$ (Figure 13a) is negligible but not at $\lambda / a=30$ (Figure $13 b$ ).

The scattered coefficients of the diffraction problem, i.e., the solution vector $A_{m n}$ of the system of equations in (5) are shown in Table 1 for an incident wave with propagation direction $\beta=0$ and wavelength $\lambda / a=5$. Results have been obtained with the interaction theory (using meshes in Figures $2 \mathrm{a}$ and $2 \mathrm{~b}$ ) and with the semi-analytical solution of [15]. A very good agreement between them can be observed. The scattered coefficients are required to calculate the excitation forces (not shown in this paper).

The interaction theory accelerates the computation of both the excitation forces and the radiation coefficients when compared to direct calculations using standard BEM software. It has been applied to an array of 20 truncated vertical cylinders representing an array of cylindrical WECs or a generic multibody 


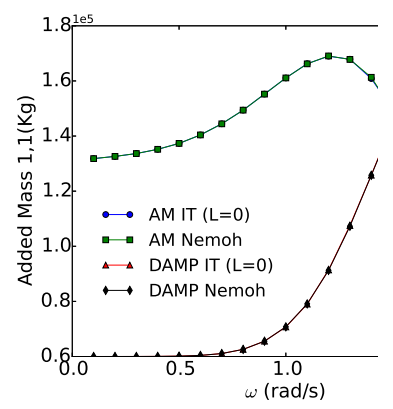

(a) Separation distance: $d$

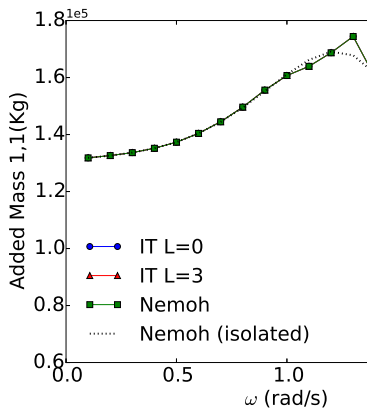

(b) Separation distance: $d$

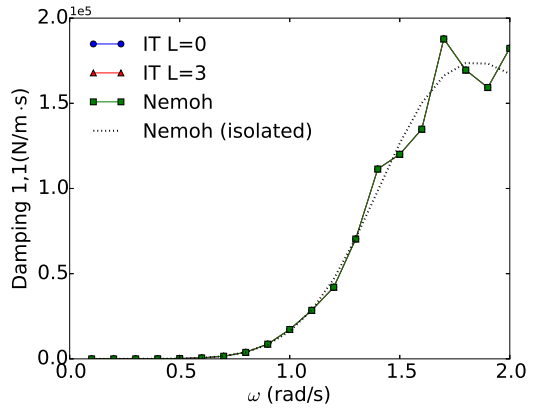

(c) Separation distance: $d / a=10$.

FIGURE 10: Added mass and damping coefficients for the first truncated vertical cylinder ( $3 m$ radius, $6 m$ draft in a $50 m$ water depth) in the two body array (Figure 11). First index corresponds to the direction of the force and the second to the degree of freedom.
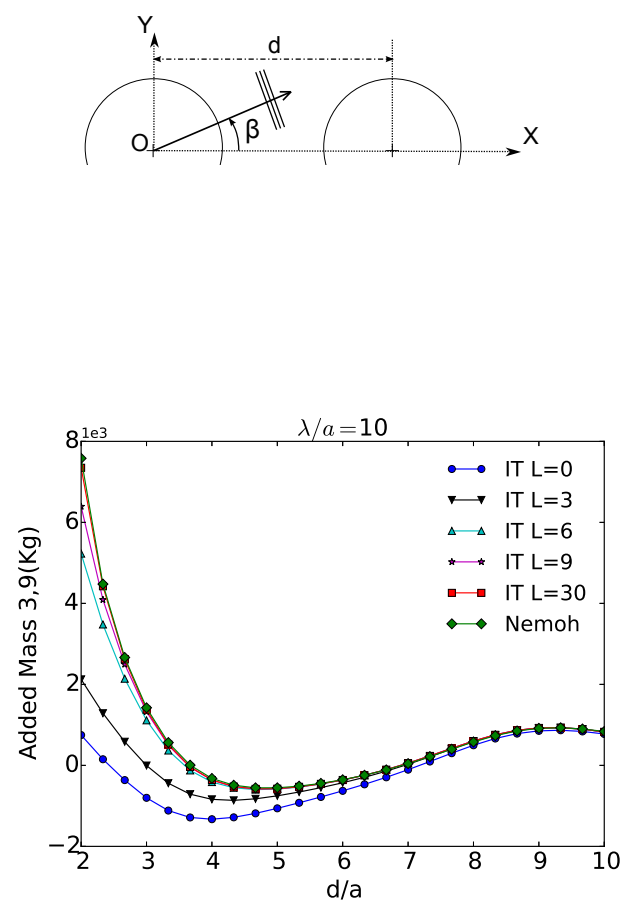

FIGURE 12: Heave-heave coupling added mass coefficient as a function of the evanescent modes truncation $L$.

type WEC such as the Wavestar. First, the scattered coefficients for both a diffraction and a radiation problem have been obtained by solving the system of equations in (5) using the appropriate incident partial wave coefficients for each case. Then, expressions in (2) have been used to reconstruct the velocity potential in the fluid domain and to calculate the free surface elevation $\left(\eta=-\left.\frac{1}{g} \frac{\partial \phi}{\partial t}\right|_{z=0}\right)$ which is shown in Figures $14 \mathrm{a}$ and $14 \mathrm{~b}$ for the diffraction and the radiation problem respectively.

\section{CONCLUSION}

The calculation of the Diffraction Transfer Matrix and the Radiation Characteristics has been implemented in the open source BEM solver NEMOH using the methodology of [2]. Results of the hydrodynamic operators of a truncated vertical cylinder and a box have been contrasted with the methodology developed by [3] and a very good agreement has been found. In addition, the DTM and RC of the cylinder have been checked against the semi-analytical solution by [15] and a very good match has been obtained.

The computation of the DTM and the RC by NEMOH facilitates the use of the finite-depth interaction theory by [1]. Results computed with this theory for a small array of two truncated vertical cylinders have been compared to direct computations using NEMOH and a very good match has been observed. The importance of evanescent modes for cases where the bodies are separated by short distances has been shown. In addition, scattered partial waves coefficients for the diffraction case have been compared to the semi-analytical solution by [15] and a very good agreement has been found.

The application of the interaction theory to an array of 20 truncated vertical cylinders has been shown to exemplify the use of the technique to compute excitation forces and radiation coefficients for large arrays of floating bodies. On-going work aimed at optimizing a generic multi-body WEC will be presented in a future paper.

\section{ACKNOWLEDGEMENTS}

The research leading to these results is part of the OceaNET project, which has received funding from the European Union's Seventh Framework Programme for research, technological development and demonstration under grant agreement no 607656. 

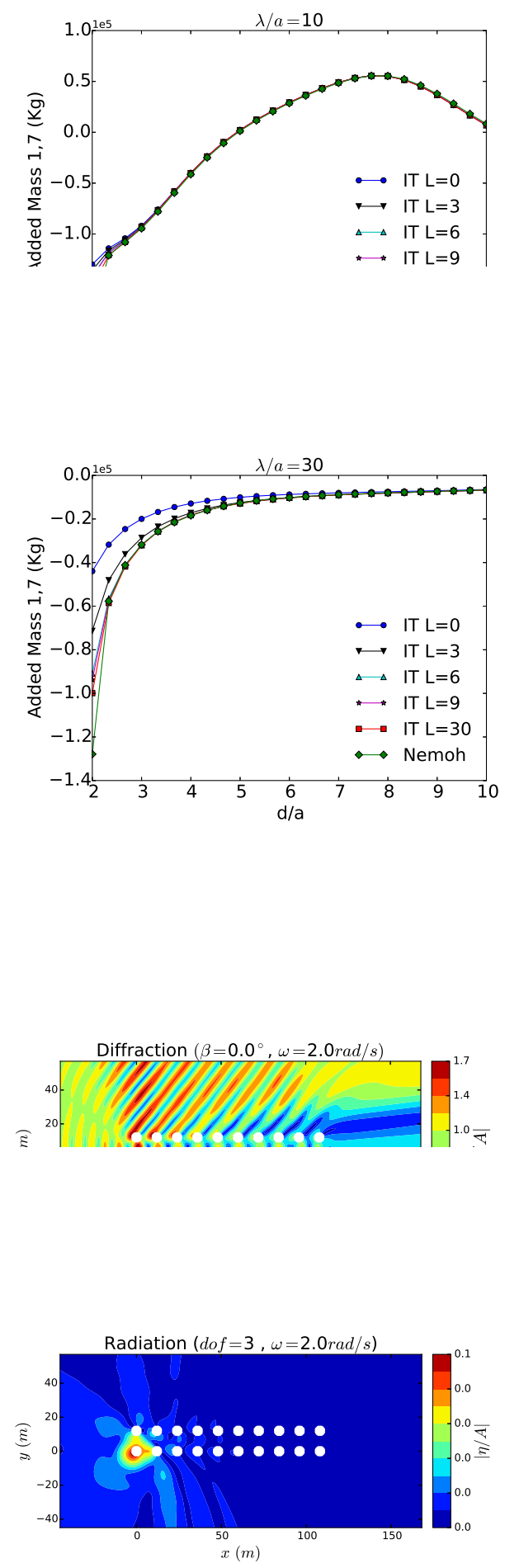

(b)

FIGURE 14: Plots of normalized free surface elevation for an array of 20 cylinders ( $3 m$ radius, $6 m$ draft, $50 m$ depth). Degree of freedom ( $\mathrm{dof}=3$ ) corresponds to the bottom left cylinder heave motion.

\section{References}

[1] Kagemoto, H., and Yue, D. K. P., 1986. "Interactions among multiple three-dimensional bodies in water waves: an exact algebraic method". Journal of Fluid Mechanics, 166(-1), Apr., p. 189.

[2] Goo, J.-S., and Yoshida, K., 1990. "A Numerical Method for Huge Semisubmersible Responses in Waves". Society of Naval Architects and Marine Engineers, 98, pp. 365387.

[3] McNatt, J. C., Venugopal, V., and Forehand, D., 2015. "A novel method for deriving the diffraction transfer matrix and its application to multi-body interactions in water waves”. Ocean Engineering, 94, Jan., pp. 173-185.

[4] Chakrabarti, S., 2000. "Hydrodynamic interaction forces on multi-moduled structures". Ocean Engineering, 27(10), Oct., pp. 1037-1063.

[5] Chakrabarti, S., 2001. "Response due to moored multiple structure interaction". Marine Structures, 14(1-2), Jan., pp. 231-258.

[6] Peter, M. a., and Meylan, M. H., 2004. "Infinite-depth interaction theory for arbitrary floating bodies applied to wave forcing of ice floes". Journal of Fluid Mechanics, 500, Jan., pp. 145-167.

[7] Kashiwagi, M. K. U., 2000. "Hydrodynamic interactions among a great number of columns supporting a very large flexible structure". Journal of Fluids and Structures, 14, pp. 1013-1034.

[8] Newman, J. N., 1999. Marine Hydrodynamics, 9th ed. The Massachusetts Institute of Technology.

[9] A.Martin, P., 2006. Multiple Scattering: Interaction of Time-Harmonic Waves with N Obstacles.

[10] McNatt, J. C., Venugopal, V., and Forehand, D., 2013. "The cylindrical wave field of wave energy converters". International Journal of Marine Energy, 3-4, Dec., pp. e26-e39.

[11] Child, B., 2011. "On the configuration of arrays of floating wave energy converters". PhD thesis, The University of Edinburgh.

[12] Fenton, J., 1978. "Wave forces on vertical bodies of revolution". Journal of Fluid Mechanics, 85, pp. 241-255.

[13] Babarit, A., and Delhommeau, G., 2015. "Theoretical and numerical aspects of the open source BEM solver NEMOH". In Proceedings of the 11th European Wave and Tidal Energy Conference 6-11th Sept 2015, Nantes, France, pp. 1-12.

[14] Abramowitz, M., and Segun A., I., 1964. Handbook of mathematical functions with Formulas, Graphs, and Mathematical Tables, first ed. Dover Publications, Inc.

[15] Zeng, X.-h., and Tang, S.-y., 2013. "The hydrodynamic interactions of an array of truncated circular cylinders as each cylinder oscillates independently with different prescribed modes". Journal of Hydrodynamics, Ser. B, 25(1), Feb., pp. 27-38. 